Leo Lahti, Samuel Myllykangas, Sakari Knuutila, and Samuel Kaski: Dependency detection with similarity constraints. Proceedigns of the MLSP'09 IEEE International Workshop on Machine Learning for Signal Processing. Grenoble, France, September 2-4, 2009.

(C)2009 IEEE. Personal use of this material is permitted. However, permission to reprint/republish this material for advertising or promotional purposes or for creating new collective works for resale or redistribution to servers or lists, or to reuse any copyrighted component of this work in other works must be obtained from the IEEE. 


\title{
DEPENDENCY DETECTION WITH SIMILARITY CONSTRAINTS
}

\author{
Leo Lahti ${ }^{1,2}$, Samuel Myllykangas ${ }^{3}$, Sakari Knuutila ${ }^{2}$ and Samuel Kaski ${ }^{1}$ \\ 1. Helsinki University of Technology, Department of Information and Computer Science \\ PO Box 5400, FI-02015 TKK, Finland \\ 2. University of Helsinki and Helsinki University Central Hospital \\ Haartman Institute and HUSLAB, Department of Pathology, Helsinki, Finland \\ 3. Stanford University School of Medicine, Department of Medicine, Division of Oncology, and \\ Stanford Genome Technology Center, Stanford University, Stanford, USA
}

\begin{abstract}
Unsupervised two-view learning, or detection of dependencies between two paired data sets, is typically done by some variant of canonical correlation analysis (CCA). CCA searches for a linear projection for each view, such that the correlations between the projections are maximized. The solution is invariant to any linear transformation of either or both of the views; for tasks with small sample size such flexibility implies overfitting, which is even worse for more flexible nonparametric or kernel-based dependency discovery methods. We develop variants which reduce the degrees of freedom by assuming constraints on similarity of the projections in the two views. A particular example is provided by a cancer gene discovery application where chromosomal distance affects the dependencies between gene copy number and activity levels. Similarity constraints are shown to improve detection performance of known cancer genes.
\end{abstract}

\section{INTRODUCTION}

We develop methods for the task of detecting statistical dependencies between multiple sources of co-occurring data. The sources are assumed to share relevant common information, and additionally contain independent but unknown type of noise. The task is to discover the relevant information; both to detect and analyse or interpret it.

This is a particular type of a data fusion task, shared by multi-view learning. In multi-view learning each source is interpreted as a different view to the same items, and the task is to enhance classification performance by combining the views. Our task can be interpreted as unsupervised multi-view learning.

The project was funded by Tekes MultiBio project. LL and SK belong to the Adaptive Informatics Research Centre and Helsinki Institute for Information Technology HIIT. LL is funded by the Graduate School of Computer Science and Engineering. SK is partially supported by EU FP7 NoE PASCAL2, ICT 216886.
The traditional statistical way of finding dependencies between data sources is canonical correlation analysis, CCA, which generalizes correlation to multidimensional sources, retaining some of the nice interpretability of correlation coefficients. While the basic correlation coefficient assumes paired scalar values, canonical correlations assume paired vectorial values. The vectors are projected to scalar components before computing the correlations, using linear projections that maximize the correlations. For multidimensional data there will be many correlation coefficients; the second components are constrained to be uncorrelated with the first, and so on.

CCA is known to have two nice properties: the result is invariant to linear transformations of the data spaces, and the solution for any fixed number of components maximizes mutual information between linear projections for Gaussian data. These insights can be interpreted as motivations for generalizing using nonparametric methods $[1,2]$ and kernel CCA $[3,4]$.

The flexibility of CCA can cause overfitting problems that are specifically harmful with small sample sizes that abound in biomedical studies, for instance. When the views are high-dimensional, the completely unconstrained linear projections involve high degrees of freedom; several ways to regularize the CCA solution have been suggested to overcome some of the associated problems [5, 6, 7]. We introduce a complementary approach that is based on bringing in prior knowledge to constrain the model family.

Assuming the dimensions of the different views are not completely unrelated but instead are formed of related pairs, it makes sense to search for more constrained projections. In our application, the views are different measurements made on the same locations of the genome, and the dimensions correspond to these particular locations. Constraining the projections to be the same or at least similar in the different views will additionally enhance interpretability of the results, given that relationships between the same compo- 
nents in the two views are natural.

Correlation-based CCA has been shown to correspond to the maximum likelihood solution of a simple generative model [4], where the two views are assumed to stem from a shared Gaussian latent variable and normally distributed data-set-specific noise. This has opened up the road to probabilistic and Bayesian formulations [8,9] which make it possible to deal rigorously with uncertainty in small sample sizes and to include prior knowledge as Bayesian priors.

We suggest also a probabilistic version for constrained dependency search that provides a robust alternative for direct maximization of correlations. While the probabilistic version is slower to compute, it is the recommended choice when prior information of the types of dependency is available, or sample size is small.

The methods will be applied in a very promising application setup for knowledge discovery with dependency detection. The task is to find potential cancer genes by studying the relationship between changes caused by cancer in gene expression and gene copy numbers, that is, amplifications or deletions caused by mutations in cancer samples. Copy number changes are a key mechanism for cancer, and combination of copy number information with gene expression measurements can reveal functional effects of the mutations; gene expression data is informative of gene activity. The rationale goes as follows: Mutations having no functional effect will not cause cancer, and cancer-related gene expression changes may be side effects. Gene expression changes caused by mutations would be strong candidates for cancer mechanisms, and they contribute to the dependencies between the two data sources. While causation can be difficult to grasp, study of the dependencies can provide an efficient proxy for such effects.

\section{CANONICAL CORRELATIONS WITH SIMILARITY CONSTRAINTS}

\subsection{Correlation-based approach}

Correlation-based CCA searches for a maximally correlated linear projection of the original data sets with paired samples $X$ and $Y$. It maximizes the correlation between the projections, $\operatorname{cor}\left(X \mathbf{v}_{x}, Y \mathbf{v}_{y}\right)$, with respect to arbitrary projection vectors $\mathbf{v}_{x}, \mathbf{v}_{y}$. However, this flexibility easily leads to overfitting as demonstrated by the case study in Section 3 .

In many applications prior information of the potential relationships between the features of the investigated data sets is available. Constraining the projections accordingly can potentially reduce overfitting and help to focus on specific types of dependencies between the two data sets. A particular example of such a model is provided by our cancer gene discovery application, where gene copy number changes are systematically correlated with the gene expression measurements from the same genes.
The relationship between the projections can be parametrized with a transformation matrix $T$ such that $\mathbf{v}_{y}=$ $T \mathbf{v}_{x}$. Maximization of the correlations between the projections leads to the following optimization problem:

$$
\arg \max _{\mathbf{v}, T}=\frac{\mathbf{v}^{T} \tilde{\Sigma}_{x y} T \mathbf{v}}{\sqrt{\mathbf{v}^{T} \tilde{\Sigma}_{x x} \mathbf{v}} \sqrt{(T \mathbf{v})^{T} \tilde{\Sigma}_{y y} T \mathbf{v}}},
$$

where the observed covariances of the two data sets are denoted by the $\tilde{\Sigma}$. Constraints on $T$ can be used to guide the dependency search. We refer to this model as Similarityconstrained CCA (SimCCA). Suitable constraints depend on the particular applications; the solutions can be made to prefer particular types of dependencies in a soft manner with an appropriate penalty term on $T$.

While we consider only one-dimensional projections in the case study, multidimensional projection matrices are also possible. The optimal projection vectors can be sought iteratively as in ordinary CCA. Direct optimization of the correlations provides a simple and computationally efficient way to detect dependencies between data sources but it lacks an explicit model to deal with the uncertainty in the data and model parameters.

\subsection{Probabilistic approach}

An explicit model-based approach for the dependency exploration task is provided by the probabilistic modeling framework. We derive a probabilistic approach which should be more robust to small sample sizes. The correlation-based CCA has a direct connection to the maximum likelihood (ML) solution of the generative model $[4,10]$ :

$$
\begin{aligned}
& X \sim N\left(W_{x} \mathbf{z}, \Psi_{x}\right) \\
& Y \sim N\left(W_{y} \mathbf{z}, \Psi_{y}\right),
\end{aligned}
$$

assuming normally distributed $\mathbf{z}$, and data-set-specific covariances $\Psi_{x}, \Psi_{y}$. The dependency between the data sets is captured by the shared latent variable $\mathbf{z}$, and $W_{x}, W_{y}$ characterize the relationship between the data sets. The covariances $\Psi_{x}, \Psi_{y}$ characterize data set-specific effects. Note that while optimal projections $\mathbf{v}$ in the correlation-based CCA (Eq. 1) operate on the observed data, the parameters of interest, $W_{x}, W_{y}$, in probabilistic CCA mediate transformations of the latent variable $\mathbf{z}$.

The solutions of the probabilistic CCA can be constrained analogously to the correlation-based approach in Eq (1), by extending the formulation to include appropriate prior terms. The joint likelihood of the model is given by

$$
\begin{aligned}
P(X, Y, W, \Psi) & \\
\sim & P\left(X, Y \mid W_{x}, W_{y}, \Psi\right) P\left(W_{y} \mid W_{x}\right) P\left(W_{x}\right) P(\Psi) \\
= & \int P\left(X, Y \mid W_{x}, W_{y}, \Psi, \mathbf{z}\right) \\
& P\left(W_{y} \mid W_{x}\right) P\left(W_{x}\right) P(\Psi) P(\mathbf{z}) d \mathbf{z} .
\end{aligned}
$$


Here $\Psi$ denotes the block-diagonal matrix of $\Psi_{x}$ and $\Psi_{y}$. While incorporation of prior information of the data setspecific effects through the $W_{x}$ and $\Psi$ provides promising lines for further work, we focus on the shared latent variables as a probabilistic alternative to the correlation-based SimCCA. The relation between the transformation matrices for the shared latent variable is encoded by the prior term $P\left(W_{y} \mid W_{x}\right)$ and can be parametrized with a transformation matrix $T$ such that $W_{y}=T W_{x}$. Assuming invertible $W_{x}^{T} W_{x}$, we have $T=W_{y}\left(W_{x}^{T} W_{x}\right)^{-1} W_{x}^{T}$.

By setting a prior on $T$ it is possible to emphasize certain types of dependencies. With unconstrained $T$ the solution reduces to ordinary probabilistic CCA. In the other extreme $T$ is an identity matrix, $T=I$, and the two shared components, derived from $x$ and $y$ respectively, would be identical. The formulation would also allow tuning of $T$ between these two extremes.

We consider the following simple prior for $T: P(T)=$ $N_{+}\left(\|(T-I)\| \mid 0, \sigma_{T}^{2}\right)=N_{+}\left(\| W_{y}\left(W_{x}^{T} W_{x}\right)^{-1} W_{x}^{T}\right)-$ $\left.I \| \mid 0, \sigma_{T}^{2}\right)$. This can be plugged into $P\left(W_{y} \mid W_{x}\right)$ in Eq. (3). We have used Frobenius norm, and $N_{+}$refers to truncated normal distribution for positive input values.

The $\sigma_{T}^{2}$ can tune the deviation of $T$ from the identity matrix; a strict version of probabilistic SimCCA (pSimCCA) is obtained with $\sigma_{T}^{2} \rightarrow 0$, while $\sigma_{T}^{2} \rightarrow \infty$ yields ordinary probabilistic CCA (pCCA). With uninformative priors $P(W), P(\Psi) \sim 1$ and normally distributed shared latent variable $\mathbf{z} \sim N(0, I)$, the model has the negative loglikelihood

$$
-\log P(X, Y, W, \Psi) \sim \log |\Sigma|+\operatorname{tr} \Sigma^{-1} \tilde{\Sigma}+\frac{\|T-I\|}{\hat{\sigma}_{T}^{2}} .
$$

Here $\Sigma=W W^{T}+\Psi$ contains the matrices $W_{x}, W_{y}$ and data set specific covariances $\Psi_{x}, \Psi_{y}$. We have added the prior for $T$, which tunes the relationship between $W_{y}$ and $W_{x}$. For other details, see $[4,5]$.

\section{ANALYSIS OF FUNCTIONAL COPY NUMBER CHANGES IN GASTRIC CANCER}

A promising biomedical application highlights the potential practical value of our approach. Constraints on the potential dependencies between gene expression and copy number are shown to improve the detection of known cancer genes. The advantages of constrained and probabilistic versions become particularly salient when the dimensionality increases and ordinary correlation-based CCA seriously overfits to the data.

\subsection{Background and motivation}

Copy number changes in chromosomal regions with tumorsuppressor or other cancer-associated genes have important contribution to cancer development and progression. Chromosomal gains and losses are likely to be positively correlated with the expression levels of the affected genes; copy number gain is likely to increase the expression of some of the associated genes whereas deletion will block gene expression. Identification of cancer-associated regions with functional copy number changes has potential diagnostic, prognostic and clinical impact for cancer studies.

Canonical correlations provide a principled framework for detecting the shared variation in gene expression and copy number data. Systematic copy number changes in a particular chromosomal region are captured by multiple copy number probes, and this is also visible in the expression levels of the genes within the affected region. The dependent signals can be subtle, however, as gene expression and copy number data are affected by high levels of unrelated biological and measurement variation, and the sample sizes are typically small.

Both correlation-based and probabilistic SimCCA combine power over the adjacent genes by capturing the strongest shared signal in gene expression and copy number observations. They can also ignore unrelated signal from poorly performing probes, or probes that measure genes that are not functionally affected by the copy number change. This provides tools to distinguish between so-called driver mutations having functional effects from less active passenger mutations, which is an important task in cancer studies. A further advantage of the probabilistic formulation is that the shared latent variable $\mathbf{z}$ provides a robust measure of the amplification effects in each patient.

\subsection{Implementation}

SimCCA is used to study the association between gene expression and copy number in a gastric cancer data set with 41 patients and 10 controls [11]. The gene expression and copy number data sets were matched for the analysis such that the closest probe by genomic location in gene expression data was selected for each copy number probe, and probes with no match between gene expression and copy number within $5000 \mathrm{bp}$ interval were discarded. The preprocessed data has gene expression and copy number measurements from 5596 genes from $\sim 700$ chromosomal regions (cytobands). To satisfy the normality assumptions of our model, the data was $\log _{2}$-transformed and the mean of the signals for each probe was set to 0 before the analysis.

Ordinary and constrained versions of canonical correlation analysis, CCA/SimCCA, were applied to investigate the dependencies between gene expression and copy numbers. The correlations were computed within a specific chromosomal window around each gene. The observed correlations provide a measure of dependency between gene copy number and expression data for each window, or chromosomal region. 

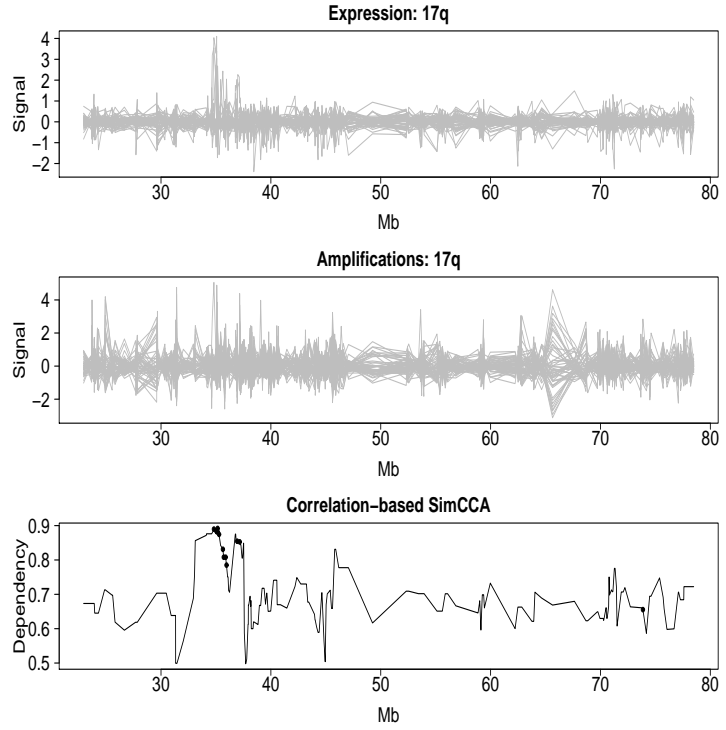

Fig. 1. Gene expression, copy number signal, and the dependency score for a sliding window of 15 genes along the chromosome arm $17 \mathrm{q}$ from the SimCCA method of Eq. (1). Known gastric-cancer associated genes from an expert-curated list are marked with black dots.

With unconstrained $T$, the models defined by Eqs. (1) and (7) reduce to ordinary correlation-based and probabilistic CCA, respectively. We assume that the constraints for $T$ are provided prior to analysis, i.e. the prior parameter $\sigma_{T}$ is fixed. Alternatively, $\sigma_{T}$ could be optimized based on external criteria such as identification of the known cancer genes in our application. Our empirical results show, however, that already a simple prior for $T$ without an explicit optimization procedure can improve the detection of known cancer genes.

We consider here the two extreme cases of the model where $\mathrm{T}$ is (i) completely unconstrained (ordinary CCA; $\left.\sigma_{T}=\infty\right)$, and (ii) $T=I\left(\sigma_{T}=0\right)$. Point estimates for the model parameters were estimated with EM algorithm in the probabilistic version. Strength of the shared signal versus marginal effects is measured with $\operatorname{Tr}\left(W W^{T}\right) / \operatorname{Tr}(\Psi)$, where $\operatorname{Tr}$ denotes matrix trace. This yields a dependency score between copy number and expression data for the investigated chromosomal neighborghood around each gene. High scores highlight regions where the dependent signal between the two data sets is particularly high relative to the data-set-specific variation.

In addition to the correlation-based and probabilistic SimCCA, we tested a simplified probabilistic version with onedimensional shared component $\mathbf{z}$ and isotropic covariances for the data-set-specific effects: $\left(\Psi_{x}=\sigma_{x}^{2} I ; \Psi_{y}=\sigma_{y}^{2} I\right)$. This is a special case of the full probabilistic model, and it reduces to principal component analysis (PCA) for con- catenated data $(X, Y)$. We refer to this method as pSimPCA. The simplified model does not distinguish between the shared and marginal effects as effectively as the full probabilistic CCA but it has fewer model parameters. Lowdimensional latent models are also faster to compute, and interpretation of the results is potentially more straightforward.

\subsection{Validation}

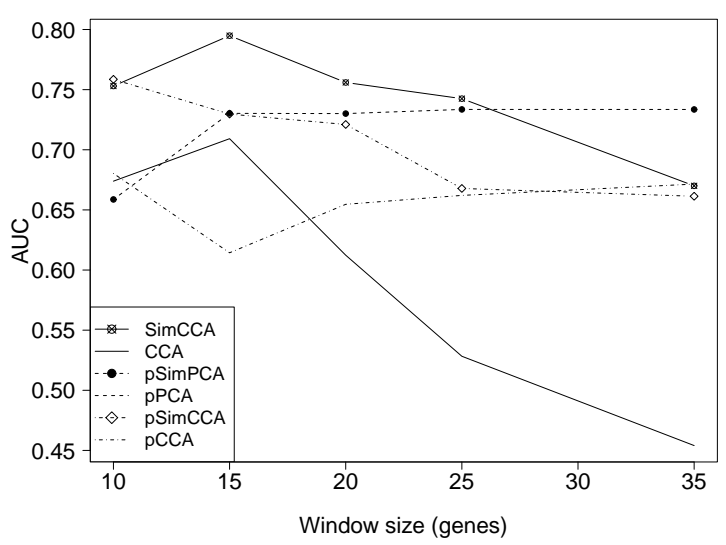

Fig. 2. AUC comparison.

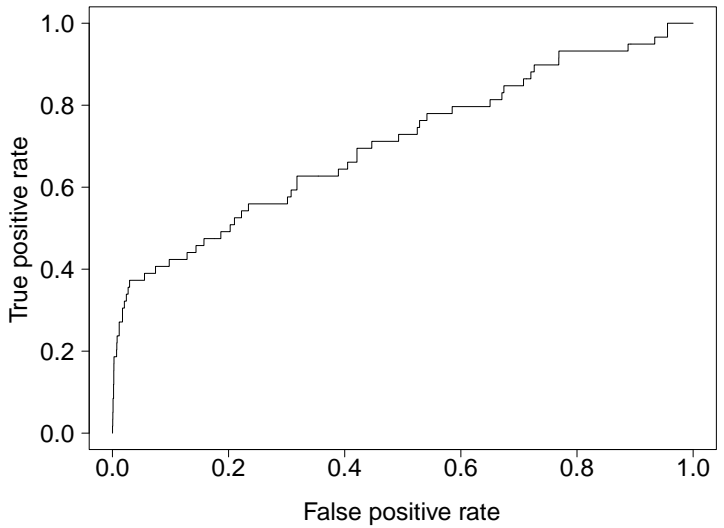

Fig. 3. ROC curve for the results from correlation-based SimCCA with a 15 -gene sliding window.

Results from the correlation-based SimCCA are illustrated for chromosome arm 17q in Fig. 1, where SimCCA highlights a known cancer-associated region. The Figure shows the dependency score for the correlation-based SimCCA with a sliding window of 15 genes genes along the chromosome arm. The correlation-based and probabilistic approaches were compared in various window sizes $(10$, 
$15,20,25$, and 35 genes). In each experiment, the gene list ordered by the dependency measure was compared to an expert-curated list of 59 gastric-cancer associated genes in our investigated data set [11].

The correlation-based and probabilistic models were compared with respect to their ability to detect the known cancer genes, measured with the AUC value of the ROC curve for each method. Results are summarized in Fig. 2. The best AUC value (0.79) was obtained with a chromosomal window of 15 genes for the correlation-based SimCCA that directly maximizes the correlations assuming identical projections (Eq. (1)). The corresponding ROC curve is shown in Fig. 3 and presents the tradeoff between true and 'false' positive findings along the ordered gene list. While a large proportion of the most significant findings are in fact known cancer genes, the remaining findings with no known associations to gastric cancer are promising candidates for further studies; among the 100 genes with highest dependencies between gene expression and copy number in their chromosomal neighborghood, $30 \%$ of the corresponding regions had previously known association with gastric cancer, while the proportion in the whole data set is $5 \%$.

The constrained dependency detection methods introduced in this paper outperformed the unconstrained models in most cases. The improved detection performance of the constrained models is likely explained by their ability to reduce overfitting. Interestingly, the most constrained probabilistic model, pSimPCA, outperforms the other approaches in the highest-dimensional case. In contrast, the performance of correlation-based CCA decreases steadily with increasing dimensionality (window size) as the number of samples (patients) remains fixed to 51 .

In our particular application, gene expression and copy number are expected to have strong linear correlations in cancer-associated chromosomal regions. Correlation-based approach is therefore directly suited for the cancer gene detection task and it has also fewer parameters than the probabilistic versions. However, the performance of correlationbased SimCCA reduces with increasing dimensionality. A likely explanation is that the correlation-based version models also some of the data set-specific effects, which is emphasized in higher-dimensions. The probabilistic formulations provide an alternative way to bring in prior knowledge of the relationships in a principled framework. A potential advantage of the probabilistic approaches is that they have an explicit model for distinguishing the shared signal from data set-specific variation.

\subsection{Biomedical interpretation of the findings}

The results obtained using the SimCCA algorithm are in general concordant with the output from signal-to-noise statistics and random permutation method that was applied previously to analyze the same data $[11,12]$. The advantage of the current method is that it combines the signal across adjacent genes within a particular chromosomal region already in the modeling step. Probabilistic SimCCA estimates the strongest shared signal between the data sets and ignores other variation using explicit modeling assumptions. Probabilistic versions also provide a measure of the amplification effect for each patient which allows robust identification of small patient groups with profound amplification effects that would be missed in previous permutation-based tests due to low event frequency.

In concordance with the previous analyses, the chromosomal area showing the most significant correlation between the gene copy number and expression was 17q12-q21 (Fig. 1). There are a number of potential target genes in that region, including $E R B B 2$ and $P P P 1 R 1 B$, which show clinical and biological relevance. The ERBB2 gene encodes a transmembrane tyrosine kinase receptor, which is a target of Herceptin. This monoclonal antibody specifically inactivates the overexpressed $E R B B 2$ protein and is used to treat metastatic breast cancer patients. The expression of $P P P 1 R 1 B$ has been shown to be associated with repression of programmed cell death and increase the survival of the cancer cells in upper gastrointestinal tract cancers [13].

Another genomic region with correlated gene copy number and expression changes is $10 \mathrm{q} 26$, and FGFR2 was identified as one of the putative target genes of that region. It was recently shown that in a set of gastric cancer cell lines, FGFR2 amplification is driving the cell proliferation and promoting cancer cell survival. Furthermore, inhibition of the FGFR2 protein by small molecules retained the growth arresting and apoptotically active phenotype [14]. The detected 1q22 region harbors the $M U C 1$ gene, whose expression was shown to be associated with the intestinal subtype of gastric cancer [11]. The 20q is one of the most frequently amplified chromosomal regions in gastric cancer. However, despite of high frequency of the amplifications the target genes in that area remain to be described. Our analysis pinpointed the strongest correlating loci to $20 \mathrm{q} 13.12$ and significantly narrow the list of putative target genes.

Some of the detected chromosomal regions did not have known association with gastric cancer; we are currently investigating these results more closely. The current application shows promising performance in detecting functional copy number changes, but biomedical studies provide also a number of other potential applications. For example, an increasing number of paired data sets are available in the future for studying the relationships between methylation, single-nucleotide polymorphisms, miRNAs, and other genomic features. 


\section{DISCUSSION}

We have introduced methods that regularize CCA solutions by taking into account similarity constraints. The methods assume that the dependencies between the different views are visible in the same dimensions, that is, the projection matrices are similar. We introduced the constraints to standard CCA, resulting in a quick method that helps in solving the "small $n$ large $p$ problem", where $n$ is the number of samples and $p$ their dimensionality.

If $n$ is very small compared to $p$, even the constrained CCA may not be sufficient, and we introduced a Bayesian variant into which further prior knowledge can be easily inserted, and which is capable of rigorously handling uncertainty in the data. While we only compare SimCCA and CCA in the present work, the probabilistic formulation allows smooth tradeoff between these two extremes, which is potentially useful in many applications.

Importantly, the constrained approaches for dependency detection can be directly applied in practical tasks in knowledge discovery; good results were obtained in a promising medical application on searching for potential cancer genes by detecting dependencies between gene expression and DNA copy number changes of the genes.

\section{REFERENCES}

[1] J.W. Fisher III, T. Darrell, W.T. Freeman, and P.A. Viola, "Learning joint statistical models for audio-visual fusion and segregation," in Advances in Neural Information Processing Systems 13, Cambridge, MA, 2000, pp. 772-778, MIT Press.

[2] A. Klami and S. Kaski, "Non-parametric dependent components," in Proceedings of ICASSP'05, IEEE International Conference on Acoustics, Speech, and Signal Processing, pp. V-209-V-212. IEEE, 2005.

[3] C. Fyfe and P.L. Lai, "ICA using kernel canonical correlation analysis.," in Proceedings of the International Workshop on Independent Component Analysis and Blind Signal Separation (ICA 2000), 2000, pp. 279-284.

[4] F.R. Bach and M.I. Jordan, "A probabilistic interpretation of canonical correlation analysis," Tech. Rep. 688, Department of Statistics, University of California, Berkeley, 2005.

[5] T. De Bie and B. De Moor, "On the regularization of canonical correlation analysis," in Proceedings of the International Conference on Independent Component Analysis and Blind Source Separation (ICA2003), S.I. Amari, A. Cichocki, S. Makino, and N. Murata, Eds. 2003.
[6] L. Sun, S. Ji, and J. Ye, "A least squares formulation for canonical correlation analysis," in ICML '08: Proceedings of the 25th international conference on Machine learning, New York, NY, USA, 2008, pp. 10241031, ACM.

[7] H.D. Vinod, "Canonical ridge and the econometrics of joint production," J. Econometrics, vol. 4, no. 2, pp. 147-166, 1976.

[8] A. Klami and S. Kaski, "Generative models that discover dependencies between data sets," in $M a$ chine learning for signal processing XVI, S. McLoone, T. Adali, J. Larsen, M. Van Hulle, A. Rogers, and S.C. Douglas, Eds., pp. 123-128. IEEE, 2006.

[9] A. Klami and S. Kaski, "Local dependent components," in Proceedings of ICML 2007, the 24th International Conference on Machine Learning, Zoubin Ghahramani, Ed., pp. 425-432. Omnipress, 2007.

[10] C. Archambeau, N. Delannay, and M. Verleysen, "Robust probabilistic projections," in Proceedings of the 23rd International conference on machine learning, W.W. Cohen and A. Moore, Eds. 2006, pp. 33-40, ACM.

[11] S. Myllykangas, S. Junnila, A. Kokkola, R.Autio, I. Scheinin, T. Kiviluoto, M.L. Karjalainen-Lindsberg, J. Hollmén, S. Knuutila, P. Puolakkainen, and O. Monni, "Integrated gene copy number and expression microarray analysis of gastric cancer highlights potential target genes.," Int J Cancer, vol. 123, no. 4, pp. 817-25, 2008.

[12] S. Hautaniemi, M. Ringnér, P. Kauraniemi, R. Autio, H. Edgren, O. Yli-Harja, J. Astola, A. Kallioniemi, and O.-P. Kallioniemi, "A strategy for identifying putative causes of gene expression variation in human cancers," J Franklin Institute, vol. 341, pp. 77-88, 2004.

[13] A. Belkhiri, A. Zaika, N. Pidkovka, S. Knuutila, C. Moskaluk, and W. El-Rifai, "Darpp-32: a novel antiapoptotic gene in upper gastrointestinal carcinomas," Cancer Res, vol. 65, pp. 6583-92, 2005.

[14] K. Kunii, L. Davis, J. Gorenstein, H. Hatch, M. Yashiro, A. Di Bacco, C. Elbi, and B. Lutterbach, "FGFR2-amplified gastric cancer cell lines require FGFR2 and Erbb3 signaling for growth and survival," Cancer Res., vol. 68, no. 7, pp. 2340-8, 2008. 